\title{
The Role of Image Enhancement in Citrus Canker Disease Detection
}

\author{
K. Padmavathi \\ Research Scholar \\ Research and Development Centre \\ Bharathiar University, Coimbatore, India
}

\author{
Dr. K. Thangadurai \\ Assistant Professor and Head, \\ P.G \& Research Department of Computer Science \\ Government Arts College (Autonomous) \\ Karur, India
}

\begin{abstract}
Digital image processing is employed in numerous areas of biology to identify and analyse problems. This approach aims to use image processing techniques for citrus canker disease detection through leaf inspection. Citrus canker is a severe bacterium-based citrus plant disease. The symptoms of citrus canker disease typically occur in the leaves, branches, fruits and thorns. The leaf images show the health status of the plant and facilitate the observation and detection of the disease level at an early stage. The leaf image analysis is an essential step for the detection of numerous plant diseases.

The proposed approach consists of two stages to improve the clarity and quality of leaf images. The primary stage uses Recursively Separated Weighted Histogram Equalization (RSWHE), which improves the contrast level. The second stage removes the unwanted noise using a Median filter. This proposed approach uses these methods to improve the clarity of the images and implements these methods in lemon citrus canker disease detection.
\end{abstract}

Keywords-Lemon tree; Citrus Canker; Recursively Separated Weighted Histogram Equalization; Median Filter; Image Enhancement; Disease detection

\section{INTRODUCTION}

In India, various citrus plants are cultivated in every state. However, the geographic areas of Andhra Pradesh, Karnataka, Punjab, Assam and TamilNadu are the leading citrus-growing states. The foremost important species and varieties of citrus grown in India are the loose-jacket oranges or santras, sweet oranges or tight jacket oranges, Sathgudl oranges, musambi oranges, acid limes or Kaghzi limes, and lemon. Several species of citrus might be affected by diseases like fungi, bacteria and viruses. In recent years, the foremost severe and customary disease is citrus canker, and it is considered a serious problem where lemon is mature on a large-scale. Citrus canker is seen on leaves, twigs, branches, fruit stalks, fruits and thorns and may lead to tree death and loss of yield. Citrus canker appears as yellowish spots or halos on leaves that gradually enlarge to $2-4 \mathrm{~mm}$ dark brown pustules. Canker on the fruit does not have the yellow halo seen on leaves. This proposed approach considers lemon leaves for detection of citrus canker disease and uses two major techniques to obtain clear, high quality images. The high quality images will facilitate easy disease identification. Within the past decade, various histogram equalisation and filtering techniques have been used to increase image quality in several applications such as eye and plant diseases. The proposed approach collects or captures unhealthy leaf images from outside and uses RSWHE and a Median filter to get high quality images. These high quality images are necessary for leaf disease detection. The following figures show various stages of citrus canker on the leaves.

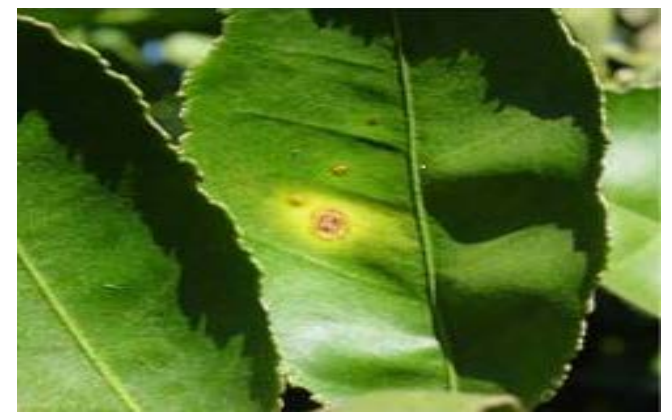

Fig. 1. Initial stage of Citrus Canker

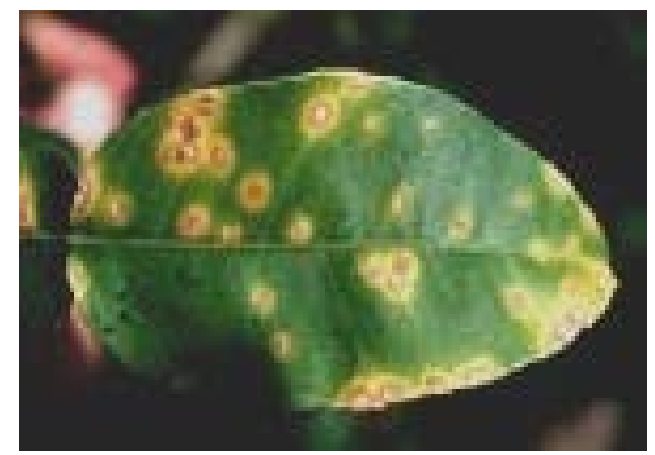

Fig. 2. Maturity stage of Citrus Canker

\section{EVAluAtion OF PROPOSED System}

Researchers have implemented various techniques to enhance the images utilised in plant disease detection. A.Camargo and J.S.Smith[2009] $]_{[1]}$ utilised histogram equalization to distribute the intensities of an image and improve the image quality and visual appearance of the plant leaf images. Anjali Naik[2010] [2] used histogram equalisation in dental disease detection to obtain high contrast images and better views of bone structure in radiographic images. T.Jintasuttisak and S. Intajag [2014] $]_{[3]}$ developed a technique called Rayleigh Contrast Limited Adaptive Histogram Equalization. Using this technique, the image contrast and overall appearance of images used to discover vision-related diseases was improved. Mary Kim and Min Gyo Chung 
[2013] $]_{[4]}$ proposed another method, Recursively Separated and Weighted Histogram Equalization (RSWHE), to enhance the image contrast and brightness preservation. RSWHE offered higher leads in comparison to earlier methods in all aspects. With RSWHE, the input histogram was segmented into more than two sub-histograms recursively using mean or median values and a weighting process was applied to modify each sub-histogram and perform histogram equalization independently. Omprakash Patel, Yogendra.P and S.Maravi and Sanjeev Sharma[2013 $]_{[5]}$ reviewed many extensions of histogram equalization. Their analysis found that RSWHE produced less mean brightness error and a high peak signal to noise ratio. This method offers higher brightness preservation and contrast enhancement that conjointly offers scalable brightness preservation owing to its recursive nature. Samuel Oporto-Díaz, Rolando, Terashima-Marín $\quad$ [2005] $]_{[6]}$ proposed a method, sequential difference of Gaussian (DoG) filters, to detect microcalcification clusters in mammograms. DoG removes unwanted noise and classifies regions. G. Kale Vaishanw[2014] $]_{[9]}$ highlighted the uses of varied filtering techniques like Gaussian, Laplacian and Median filters. He emphasised the importance of image enhancement in X-ray lung images. He maintained that the filtering technique selection is based on both the application and process image enhancement. M.A. Shaikh and Dr. S.B.Sayyad[2014 $]_{[10]}$ used colour image enhancement in the agricultural domain. They implemented histogram equalization for contrast enhancement and compared linear (Wiener) filters and nonlinear(Median) filters. They observed that the Median filter was better at noise reduction and removed the blurred effect of the image. J.M. Durge, Prof. N.P. Bobade and Dr N.N. Mhala[2015][8] proposed to detect early stage lung cancer. They used the Median filter to enhance the image and suppress noise and other fluctuations of lung images.

Based on our literature review, we carried out our image enhancement using two different methods: histogram and filtering. Our proposed approach uses histogram equalization for contrast enhancement and filtering for unwanted noise removal.

\section{PROPOSED APPROACH}

The proposed methods play an important role in citrus canker disease detection by improving image quality with greater clarity. Here, we have developed two strategies to enhance the contrast and quality of the lemon leaf images: RSWHE increases the contrast of the image, and the Median filter removes the noise.

\section{A. Mathematical Model}

1) Recursively separated and Weighted Histogram Equalization (RSWHE)

RSWHE increases the contrast of an image in the following three stages:

a. Histogram segmentation: Consider the image $X$ and calculate the histogram $\mathrm{H}(\mathrm{X}) \mathrm{H}(\mathrm{X})$ is then divided into the number of sub-histograms.

b. Histogram weighting: Change the sub-histograms using normalized power law. c. Histogram equalization: Equalize the weighted subhistograms independently over the changed subhistograms.

a) Histogram Segmentation: This divides the histogram $\mathrm{H}(\mathrm{X})$ using the recursion level $\mathrm{r}$ and creates 2r sub-histograms based on the mean value. Consider a sub-histogram $\mathrm{H}^{\mathrm{t}}(\mathrm{X})$ over a range $\left[X_{l}, X_{u}\right]$ at a recursion level $t(0 \leq t<r)$. The subhistogram $\mathrm{H}^{\mathrm{t}}(\mathrm{X})$ mean value (i.e., $\mathrm{X}_{\mathrm{m}}^{\mathrm{t}}$ ) is computed using the formula: $X_{m}^{t}=\sum_{\underline{h}=1}^{u} \underline{k} \cdot p(k) / \sum_{k}^{u}=1 p(k)$.

$\mathrm{H}^{\mathrm{t}}(\mathrm{X})$ divides into $\mathrm{H}^{\mathrm{t}+1} \mathrm{~L}(\mathrm{X})$ and $\mathrm{H}^{\mathrm{t}+1} \mathrm{U}(\mathrm{X})$ based on $\left[\mathrm{X}_{\mathrm{m}}^{\mathrm{t}}\right.$. Here, $\mathrm{H}^{\mathrm{t}+1}{ }_{\mathrm{L}}(\mathrm{X})$ and $\mathrm{H}^{\mathrm{t}+1} \mathrm{U}(\mathrm{X})$ over $\left[\mathrm{X}_{\mathrm{l}}, \mathrm{X}_{\mathrm{m}}^{\mathrm{t}}\right]$ and $\left[\mathrm{X}_{\mathrm{m}}^{\mathrm{t}}+1, \mathrm{X}_{\mathrm{u}}\right.$, respectively.

b) Histogram Weighting: The histogram segmentation creates 2 r sub-histograms $H_{i}^{r}(X)\left(0<i<=2^{r}-1\right)$. For $r=2$, the histogram weighting changes the Probability Density Function $\mathrm{p}(\mathrm{k})$ of $\mathrm{H}_{\mathrm{i}}^{\mathrm{r}}(\mathrm{X})$ as follows:

i) Calculate the maximum and minimum probability ( $p_{\max }$

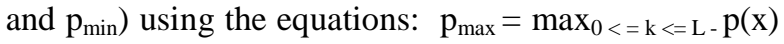
and $\mathrm{p}_{\min }=\min _{0<=\mathrm{k}<=\mathrm{L}-1} \mathrm{P}(\mathrm{x})$.

ii) Calculate a cumulative probability $\alpha_{\mathrm{i}}$ using $\alpha_{\mathrm{i}}=\sum_{\underline{k}=1}^{\mathrm{u}}=1$ $\mathrm{p}(\mathrm{k})$ for $\mathrm{H}_{\mathrm{i}}^{\mathrm{r}}(\mathrm{X})$.

iii) Change the $\mathrm{p}(\mathrm{k})$ into weighted $\mathrm{p}(\mathrm{k})$ using the following formula:

$$
\mathrm{p}_{\mathrm{w}}(\mathrm{k})=\mathrm{p}_{\max }\left(\mathrm{p}(\mathrm{k})-\mathrm{p}_{\min } / \mathrm{p}_{\max }-\mathrm{p}_{\min }\right)^{\alpha \mathrm{i}}+\beta(1<=\mathrm{k}<=\mathrm{u})
$$

Here, $\beta$ adjusts the brightness and contrast of the image and $\beta>=0$. The $\mathrm{p}_{\mathrm{w}}(\mathrm{k})$ is normalized using ${ }_{\mathrm{pwn}}(\mathrm{k})={ }_{\mathrm{pw}}(\mathrm{k}) / \sum^{\mathrm{L}-}$ $1_{\mathrm{k}=0} \mathrm{p}(\mathrm{k})$ and forwarded to the next module.

c) Histogram Equalization: The $\mathrm{p}_{\mathrm{wn}}(\mathrm{k})$ contains $2^{\mathrm{r}}$ curves and bounds by the range $\left[\mathrm{X}_{\mathrm{l}}, \mathrm{X}_{\mathrm{u}}\right]$ of $\mathrm{H}_{\mathrm{i}}{ }^{\mathrm{r}}(\mathrm{X})$. This module equalizes all $2^{\mathrm{r}}$ sub-histograms and combines all resultant images. It provides high quality images as its output.

Input

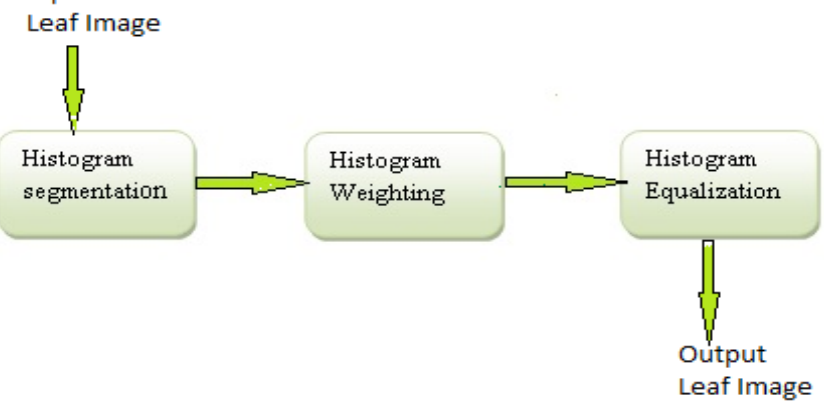

Fig. 3. A model of RSWHE

\section{2) Median Filter}

We can get the higher end in conserving, removing noise and isolating noise spikes using the Median filter. The median $\mathrm{m}$ could be a group of numbers in this group if half the numbers are less than $\mathrm{m}$ and half are greater. The median $\mathrm{m}$ could be a midpoint pixel value that is drawn from the neighbourhood sorted distribution values. It does not produce a new, unrealistic pixel value. The median operator arranges the values within the pixel element neighbourhood at each 
pixel element location. This reduces edge blurring and loss of image detail.

\section{Algorithm:}

Step 1: Select a $3 \times 3$ size two-dimensional centre window $\mathrm{p}(\mathrm{x}, \mathrm{y})$ from the input image.

Step 2: Rank the pixel values $\mathrm{p}(\mathrm{x}, \mathrm{y})$ within the selected window in ascending order and find the median, maximum and minimum pixel values (Pm, Pmax and Pmin).

Step 3: If the pixel value $\mathrm{p}(\mathrm{x}, \mathrm{y})$ has the limit $\mathrm{Pmin}<\mathrm{P}(\mathrm{x}$, y) $<$ Pmax, Pmin $>0$ and $P \max <255, \mathrm{p}(\mathrm{x}, \mathrm{y})$ is taken into account as uncorrupted. Otherwise, $\mathrm{p}(\mathrm{x}, \mathrm{y})$ is taken into account as corrupted.

Step 4: The corrupted $\mathrm{p}(\mathrm{x}, \mathrm{y})$ has two categories:

Category 1: If Pmin $<\mathrm{Pm}<\mathrm{Pmax}$ and $0<\mathrm{Pm}<255$, replace the corrupted $\mathrm{p}(\mathrm{x}, \mathrm{y})$ with $\mathrm{Pm}$.

Category2: Pmin considered a noisy pixel. Here, calculate the difference between each adjacent pixel across the ranked values, find the maximum difference and mark it as the next processed pixel.

Step 5: Apply steps 1 to 4 to the entire image until the process is complete.

Neighborhood Values:

$126,127,133,115,119,135,118,120,150$

Max: 135

Min: 115

TABLE I. MEdiAn FILTER-NEIGHBORHOOD VALUES SELECTION

\begin{tabular}{|l|l|l|l|l|}
\hline 130 & 140 & 123 & 125 & 126 \\
\hline 134 & $\mathbf{1 2 6}$ & $\mathbf{1 2 0}$ & $\mathbf{1 1 8}$ & 122 \\
\hline 124 & $\mathbf{1 2 7}$ & $\mathbf{1 5 0}$ & $\mathbf{1 3 5}$ & 118 \\
\hline 119 & $\mathbf{1 3 3}$ & $\mathbf{1 1 5}$ & $\mathbf{1 1 9}$ & 123 \\
\hline 120 & 110 & 116 & 111 & 130 \\
\hline
\end{tabular}

\section{EXPERIMENTS AND RESULTS}

Image enhancement methods have been applied to various lemon citrus canker diseased leaf images using MATLAB. RSWHE has been used to increase the brightness of images, while the Median filter has been used for de-noising corrupt images.

Here, we have taken sample citrus canker diseased leaves images and applied RSWHE and Median filter and got high quality citrus canker diseased images.

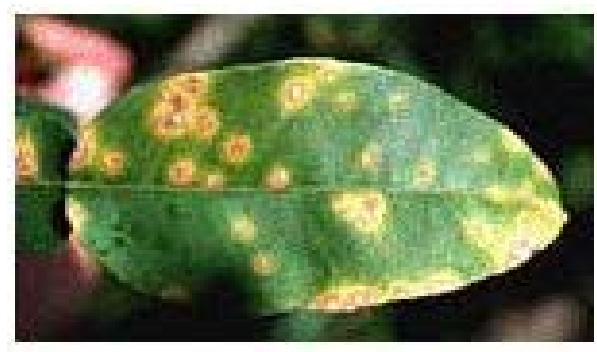

Fig. 4. Original image

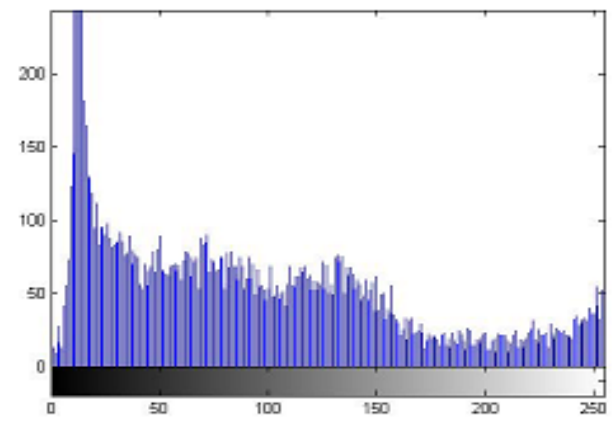

Fig. 5. Original Image Histogram

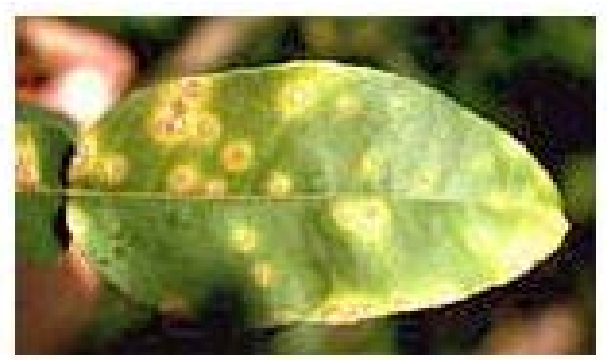

Fig. 6. Equalized Image

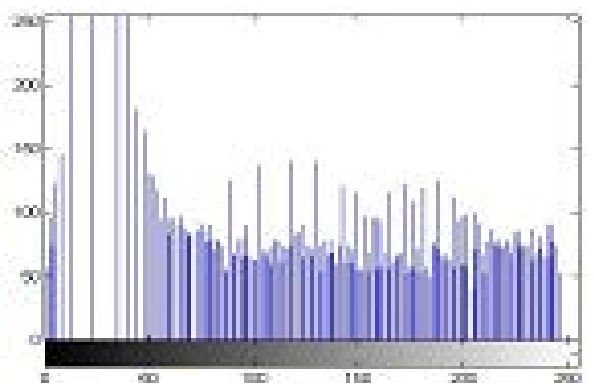

Fig. 7. Equalized Image Histogram

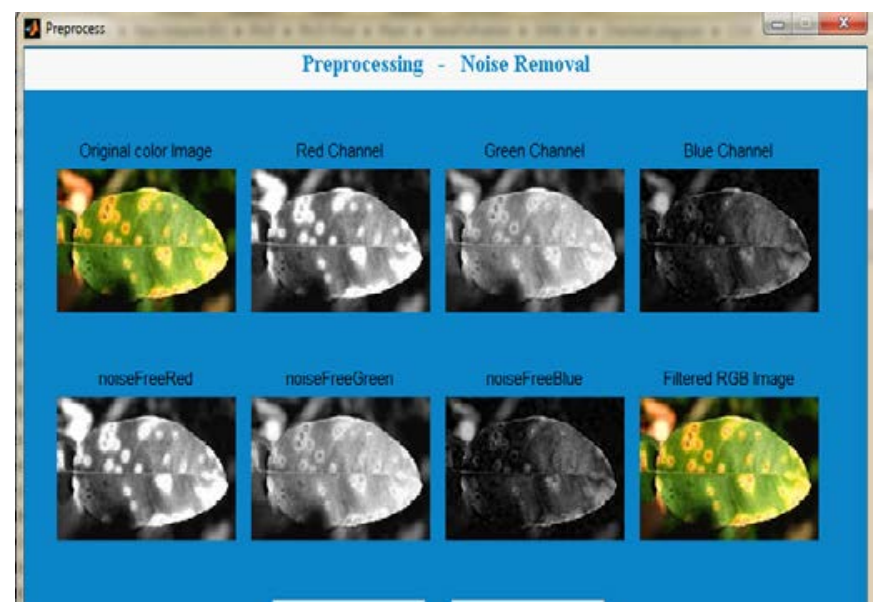

Fig. 8. Filtering process

\section{CONCLUSION}

Compared to the predominant strategies, our proposed approach has produced better image enhancement. This approach applies RSWHE and a Median filter to enhance 
citrus canker diseased leaf images. Using this method, we tend to get clear, high quality leaf images for further processing. This approach highlights the results of the enhancement process in citrus canker disease detection, showing that the visual illustration of diseased leaf images is improved.

In the future, enhanced citrus canker images will be taken and applied segmentation method which is used to extract the diseased portion. They may be utilised for citrus canker disease detection and the identification of disease level.

\section{REFERENCES}

[1] Camargo A, Smith J S. An image-processing based algorithm to automatically identify plant disease visual symptoms. Biosyst Eng 2009; 102: 9-21.

[2] Anjali Naik, Tikhe S v, Bhide S D. Histogram Equalization for ClassIdentification of Dental Disease Using Digital Radiography. Communications in Computer and Information Science 2010; 70: 144151.

[3] Jintasuttisak T, Intajag S. Color retinal image enhancement by Rayleigh contrast-limited adaptive histogram equalization. In: Control, Automation and Systems (ICCAS) $201414^{\text {th }}$ International Conference ; 22-25 October 2014; Seoul: IEEE. pp. 692-697.

[4] Kim M, Chung MG. Recursively Separated and Weighted Histogram Equalization for brightness Preservation and Contrast Enhancement. IEEE T Consum Electr 2008; 54: 1389-1397.

[5] Omprakash Patel, Yogendra P. S. Maravi and Sanjeev Sharma. A Comparative Study of Histogram Equalization Based Image Enhancement Techniques for Brightness Preservation and Contrast Enhancement. Signal \& Image Processing: An International Journal (SIPIJ) 2013; 4: 11-25.

[6] Oporto-Díaz S, Hernández-Cisneros R, Terashima-Marín H. Detection of Microcalcification Clusters in Mammograms Using a Difference of Optimized Gaussian Filters. In: Kamel M, Campilho A, editors. Image Analysis and Recognition. Toronto, Canada: Springer Berlin Heidelberg Publisher, 2005; pp: 998-1005.

[7] Swati R. Dixit, Dr.Amol Y. Deshmukh. Design Strategies for Various Edge Detection Techniques for Disease Detection. International Journal of Scientific Engineering and Applied Science 2015; 1: 498-505.

[8] Durge J M, Bobade N P, Dr.Mhala N N. Image Enhancement to Detect The Lung Cancer at Early Stage Using Median Filter. International Journal of Science, Engineering and Technology 2015; 3: 936-939.

[9] Kale Vaishanw G. X-Ray Lung Image Enhancement by Spatial filtering. In: VISHWATECH 2014 Two days National Conference; 21-22 February 2014; Maharastra, INDIA: International Journal of Innovative Research in Science, Engineering and Technology. Pp. 330-336.
[10] Shaikh M A, Sayyad S B. Color Image Enhancement Filtering Techniques for Agricultural Domain Using MATLAB. In: VIII International Symposium on Operational Remote Sensing Applications: Opportunities, Progress and Challenges; 9-2 December 2014; Hyderabad, INDIA.

[11] Gavhale KR, Gawande U, Hajari K O. Unhealthy region of citrus leaf detection using image processing techniques. In: IEEE 2014 International Conference for Convergence of Technology; 6-8 April 2014; Pune, INDIA: IEEE. Pp. 1-6.

[12] Kim D G, Burks T F, Schumann A W, Zekri M, Zhao X, Qin J. Detection of Citrus Greening Using Microscopic Imaging. Agricultural Engineering International: the CIGR Ejournal 2009; XI: 1-17.

[13] Chen E, Chung P, Chen C, Tsai H, Chang C. An Automatic Diagnostic System for CT Liver Image Classification. IEEE T Bio-Med Eng 1998; 45: 783-794.

[14] Narvekar PR, Kumbhar M M, Patil S N. Grape Leaf Diseases Detection \& Analysis using SGDM Matrix Method. International Journal of Innovative Research in Computer and Communication Engineering 2014; 2: 3365-3372.

[15] Gwan D, Burks TF, Qin J, Bulanon D M. Classification of grapefruit peel diseases using color texture feature analysis. Int J Agric \& Biol Eng 2009; 2: 41-50.

[16] Kumar V, Bansal H. Performance Evaluation of Contrast Enhancement Techniques for Digital Images. International Journal of Computer Science and Technology 2011; 2: 23-27.

[17] Huynh-The T, Le B, Lee S, Le-Tien T, Yoon Y. Using weighted dynamic range for histogram equalization to improve the image contrast. Int J Image Video Process 2014; 44.

[18] Rani S, Kumar M. Contrast Enhancement using Improved Adaptive Gamma Correction with Weighting Distribution Technique. International Journal of Computer Applications 2015; 101: 47-53.

[19] Sonia, Goel M, Goel R. Comparative Study of Image Enhancement Using Histogram Equalization Based Processing Techniques. In: ITCSE 2015 National Conference on Innovative Trends in Computer Science Engineering; 4 April 2015; Bahal, INDIA: IJRRA. pp. 172-174.

[20] Kotkar VA, Sanjay S. Gharde. Review of Various Image Contrast Enhancement Techniques. International Journal of Innovative Research in Science, Engineering and Technology 2013; 2: 2786-2793.

[21] Chen SD, Ramli A R. Contrast enhancement using recursive meanseparate histogram equalization for scalable brightness preservation. IEEE T Consum Electr 2003; 49: 1301-1309.

[22] Sim K S, Tso C P, Tan Y Y. Recursive sub-image histogram equalization applied to gray scale images. Pattern Recogn Lett 2007; 28: $1209-1221$.

[23] Gonzalez R, Woods R. Digital Image Processing. 3rd ed. Delhi, India: Pearson education, 2010.

[24] Gonzalez R, Woods R, Eddins S. Digital Image Processing using MATLAB. 3rd ed. Delhi, India: Pearson education, 2012. 